\title{
Dynamic Variations in Punicalagin and Related Metabolic Substances in Pomegranate Fruit and Leaves During Development Periods
}

\author{
Lijuan Feng, Yanlei Yin*, Xuemei Yang, Haixia Tang and Qiqing Jiao
}

Shandong Institute of Pomology, Tai'an, Shandong 271000, China

Pomegranate (Punica granatum L.) is a functional fruit that is abundant in bioactive substances. Punicalagin is an abundant and important phenolic compound in pomegranate, but the variation patterns and linkage of punicalagin and related synthetic bioactive substances during development are unclear. In this study, the dynamic variations in punicalagin and related synthetic bioactive substances were investigated in different parts of pomegranate during development phases. Punicalagin and related synthetic substances in the pomegranate pericarp, juice, seeds, and leaves were determined by high-performance liquid chromatography. Results revealed that punicalagin, gallic acid, shikimic acid, and total phenol contents, as well as DPPH radical scavenging activity, decreased gradually in the fruit, whereas pentagalloylglucose and 3-dehydroshikimic acid contents increased gradually. Seven substances yielded different variation patterns in the leaves. Ellagic acid content initially decreased and then increased in the fruit and leaves. Pentagalloylglucose was mainly detected in the leaves, while shikimic acid, 3-dehydroshikimic acid, punicalagin, gallic acid, and ellagic acid were mainly found in the pericarp. The highest punicalagin concentration was $156.735 \mathrm{mg} \cdot \mathrm{g}^{-1}$ in the TSH pericarp. Shikimic acid and 3-dehydroshikimic acid were closely related to gallic acid. Punicalagin was significantly positively correlated with gallic acid in the fruit, and significantly negatively correlated with pentagalloylglucose. Antioxidant activity and total phenol content were also closely correlated. Punicalagin and related substances presented different variation patterns in various pomegranate parts during development. Punicalagin was the most abundant phenolic compound in the pericarp, and this finding correlated significantly with gallic acid, ellagic acid, shikimic acid, 3-dehydroshikimic acid, and pentagalloylglucose in the fruit. The pomegranate pericarp contained abundant phenolic compounds and exhibited high antioxidant activity.

Key Words: antioxidant properties, bioactive substances, dynamic variation, pomegranate, punicalagin.

\section{Introduction}

Pomegranate (Punica granatum L.) is one of the oldest and most widely grown horticulture crops in several tropical and subtropical countries including India, Turkey, China, the United States, Spain, Italy, Chile, and South Africa (Fawole and Opara, 2013; Chater et al., 2018). Pomegranates are very rich sources of phenolic compounds that are mainly found in various

Received; March 14, 2019. Accepted; June 28, 2019.

First Published Online in J-STAGE on September 3, 2019.

This work was supported by the Shandong Key Research and Development Projects (2018GNC111009), Shandong Agricultural Improvement Seed Projects (2017LZN023), Agricultural Science and Technology Innovation Engineering Discipline Team of Shandong Academy of Agricultural Sciences (CXGC2018E22), Natural Science Foundation of Shandong Province (ZR2015YL056).

* Corresponding author (E-mail: yylei66@sina.com). parts such as the pericarp, juice, seeds, leaves, and flowers. For instance, pomegranate contains major phenolic compounds including ellagitannins, punicalagins, flavonoids, gallic acid, and ellagic acid (Singh et al., 2014; BenSaad et al., 2017). These pomegranate phenolic compounds have several health-promoting effects such as antioxidative, anti-inflammatory, antimicrobial, antidiabetic, and antitumor properties (Orgil et al., 2014; Modaeinama et al., 2015; Clementi et al., 2017).

Punicalagin is the most abundant and predominant ellagitannin found in pomegranate (Seeram et al., 2005; Landete, 2011). The biosynthetic pathway of other ellagitannins, such as tellimagrandin II and cornusiin $\mathrm{E}$, has been reported, but the biosynthetic pathway of punicalagin is poorly understood (Vermerris and Nicholson, 2006). Gallic acid, pentagalloylglucose, and ellagic acid are related to ellagitannin metabolism (Vermerris and Nicholson, 2006; Dewick, 2009). 
Shikimic acid and 3-dehydroshikimic acid are closely related to gallic acid metabolism (Ossipov et al., 2003; Muir et al., 2011). Gallic acid is metabolized into pentagalloylglucose via a series of enzymatic reactions (Grundhöfer et al., 2001). Pentagalloylglucose is then transformed into ellagitannins such as tellimagrandin II and cornusiin $\mathrm{E}$, which are precursors in ellagitannin metabolism (Niemetz and Gross, 2003; Dewick, 2009). Ellagic acid is a degradation product of ellagitannins such as tellimagrandin II and punicalagin (Dewick, 2009). Punicalagin belongs to the ellagitannin family, and its metabolism is similar to ellagitannin. Therefore, shikimic acid, 3-dehydroshikimic acid, gallic acid, pentagalloylglucose, and ellagic acid are possibly related to punicalagin metabolism (Qin et al., 2017) (Fig. 1).

Punicalagin in pomegranate has been isolated and identified (Fischer et al., 2011), and its content ( $\mathrm{Lu}$ et al., 2008) has been evaluated. Punicalagin has several health benefits, including antioxidant, antiviral, antiproliferation and anticancer properties, and has chemopreventive and chemotherapeutic effects against cervical cancer in humans through inhibition of the $\beta$ catenin signaling pathway (Clementi et al., 2017; Tang et al., 2017). Punicalagin inhibits lipopolyscacchrideinduced memory impairment via anti-inflammatory and

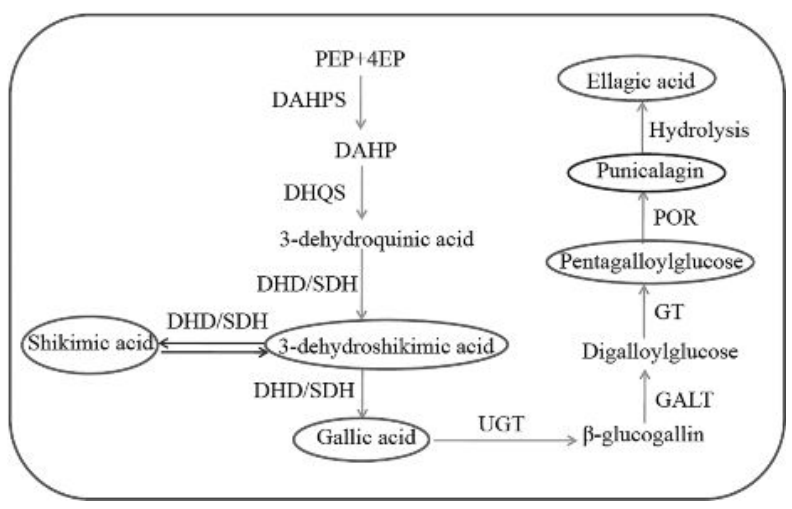

Fig. 1. The putative metabolic pathway of punicalagin (Grundhöfer et al., 2001; Dewick, 2009; Muir et al., 2011). PEP and E4P produces DAHP under the action of DAHPS. 3-dehydroquinic acid is produced from DAHP by DHQS. Under the effect of $\mathrm{DHD} / \mathrm{SDH}, 3$-dehydroquinic acid produces 3-dehydroshikimic acid; 3-dehydroshikimic acid produces shikimic acid and gallic acid. Gallic acid produces $\beta$-glucogallin under the action of UGT. Digalloylglucose is produced from $\beta$-glucogallin by GALT. Digalloylglucose generates pentagalloylglucose through a series of reactions under the action of GT. Pentagalloylglucose is metabolized into punicalagin by the effect of POR. The hydrolysis of punicalin produces ellagic acid. PEP: Phosphoenolpyruvic acid, E4P: D-erythrose-4-phosphate, DAHP: 3deoxy-D-arabinoheptulosonic acid-7-phosphates, DAHPS: 3deoxy-D-arabinoheptulosonic acid-7-phosphate synthesis, DHQS: 3-dehydroquinate synthase, DHD/SDH: Bifunctional 3dehydroquinate dehydratase/shikimate dehydrogenase, UGT: UDP-glucose: gallate glucosyltransferase, GALT: $\beta$-glucogallin O-galloyltransferase, GT: Galloyltransferase, POR: Pentagalloylglucose oxygen oxidoreductase. anti-amylogenic mechanisms through inhibition of NF$\kappa \mathrm{B}$ activation (Kim et al., 2017). However, the metabolic mechanism of punicalagin in pomegranate has not yet been investigated. Therefore, our research aimed to (i) investigate the dynamic variations and antioxidant properties of punicalagin and its related synthetic substances in pomegranate pericarp, juice, seeds, and leaves during development and (ii) identify the possible linkage between punicalagin and related synthetic bioactive substances in the fruit and leaves. This study provides a theoretical basis for the functional development of pomegranate, and can support the further development of the pomegranate industry.

\section{Materials and Methods}

\section{Sample preparation}

Two Chinese pomegranates, 'Taishanhong (TSH)' and 'TaishanSanbaitian (TSSBT)' were used as experimental materials. TSH has a red pericarp, red arils and good quality, and TSSBT has a white pericarp, white arils and excellent quality. Ten trees were randomly chosen from a pomegranate orchard located in Taian City, Shandong Province, China, where they were grown under homogeneous conditions. Fruits and functional leaves were picked at 10-day intervals from July 15 to October 2 (full maturity) in 2015-2017. The sampling dates were July 15(S1), July 25(S2), August 4(S3), August 14(S4), August 24(S5), September 3(S6), September 13(S7), September 23(S8), and October 2(S9), respectively. Samples of 20 representative fruits were collected from four tree orientations (east, west, south, and north). The samples were placed in a cooler and transported to a lab. The fruits were peeled manually with a peeler. The pomegranate arils were squeezed in two-layered muslin cloth to extract the juice completely. Fruit peels, seeds, juice, and leaves were lyophilized using an LGJ-10 freeze dryer (Songyuanhuaxing Company, Beijing, China) and then stored at $-80^{\circ} \mathrm{C}$ in a refrigerator until analysis.

\section{Extraction of punicalagin, gallic acid and ellagic acid}

The extraction method for punicalagin, gallic acid, and ellagic acid was established by Schieber et al. (2001). Frozen peels, juices, seeds, and leaves were ground in liquid nitrogen by using a mortar and pestle. A tissue sample $(1.0 \mathrm{~g})$ was added to $10 \mathrm{~mL}$ of ultrapure water. The aqueous solution was adjusted to $\mathrm{pH} 7.0$ and extracted with $10 \mathrm{~mL}$ of ethyl acetate to determine punicalagin. The extracts were shaken for $30 \mathrm{~min}$ on a shaking bed and centrifuged at $4,700 \times \mathrm{g}$ for $15 \mathrm{~min}$ at $4^{\circ} \mathrm{C}$. The upper phase containing ester was separated, and the aqueous phase was extracted twice with ethyl acetate. The ester phase, which was neutral phenol, was combined, and the combined extracts were evaporated to dryness with an RE-52AA rotary evaporator (Shanghaiyarong Biochemical Instrument Company, Shanghai, China). The residue was dissolved in metha- 
nol $(2 \mathrm{~mL})$, membrane filtered $(0.22 \mu \mathrm{m})$, and used for HPLC analysis. The aqueous phase was adjusted to $\mathrm{pH} 2.0$ and extracted with the same method used to isolate gallic acid and ellagic acid.

\section{Extraction of shikimic acid and 3-dehydroshikimic acid}

The shikimic acid and 3-dehydroshikimic acid contents of the extracts were determined according to the method described by Zhou et al. (2010). A tissue sample $(1.0 \mathrm{~g})$ was added to $40 \mathrm{~mL}$ of ultrapure water in a covered conical flask. After ultrasonic treatment for 90 min at $60^{\circ} \mathrm{C}$ with a KQ2200E-type ultrasonic clean$\mathrm{er}$, the extracts were centrifuged at $7,500 \times \mathrm{g}$ for $15 \mathrm{~min}$. The supernatant liquors were diluted with ultrapure water to $50 \mathrm{~mL}$ in a volumetric flask, filtered through a membrane $(0.22 \mu \mathrm{m})$, and stored at $-80^{\circ} \mathrm{C}$ in a refrigerator until HPLC determination.

\section{Extraction of pentagalloylglucose}

The extraction method for pentagalloylglucose was established by Xie et al. (2013). A tissue sample (0.5 g) was added to $40 \mathrm{~mL}$ methanol in a conical flask, and then ultrasonically treated for $30 \mathrm{~min}$ at $37^{\circ} \mathrm{C}$ with a KQ2200E-type ultrasonic cleaner. The extracts were centrifuged at $7,500 \times \mathrm{g}$ for $20 \mathrm{~min}$. The volume of the supernatant liquors was adjusted to $50 \mathrm{~mL}$ by adding methanol in a volumetric flask. The extracts were filtered with a $0.22 \mu \mathrm{m}$ membrane and used for HPLC determination.

\section{HPLC-DAD analysis}

HPLC analysis was conducted using an Agilent 1260 series HPLC system (1260 quaternary pump VL, series DEABJ01988) coupled with a photodiode array detector (series DEAAX01400) and an autosampler (series DEAAC23689), and operated with Agilent ChemStation. Separations were performed on a Zorbax SB-C18 column $(150 \mathrm{~mm} \times 4.6 \mathrm{~mm}$ ID, $5 \mu \mathrm{m})$. The chromatographic conditions of punicalagin and its related compounds were established by our research group (Feng et al., 2017). The mobile phases of punicalagin, gallic acid, and ellagic acid consisted of acetonitrile and glacial acetic acid $(2 \%, v / v)$, at a ratio of
20:80 (v/v). The mobile phases of shikimic acid and 3dehydroshikimic acid were composed of methanol and phosphoric acid $(1 \%, \mathrm{v} / \mathrm{v})$, at a ratio of 5:95 (v/v). The mobile phases of pentagalloylglucose were acetonitrile and $0.5 \%(\mathrm{v} / \mathrm{v})$ phosphoric acid at a ratio of 20:80 (v/v). The optimum absorption wavelength of punicalagin, gallic acid, and ellagic acid was $280 \mathrm{~nm}$ under the same conditions. The absorption wavelength of shikimic acid and 3-dehydroshikimic acid was $214 \mathrm{~nm}$, and the absorption wavelength of pentagalloylglucose was $279 \mathrm{~nm}$. The column temperature was set at $30^{\circ} \mathrm{C}$. The flow rate of the mobile phase was $0.8 \mathrm{~mL} \cdot \mathrm{min}^{-1}$. The injection volume for all samples was $10 \mu \mathrm{L}$. The regression equation, $R^{2}$, linear range, and LOD of punicalagin and related substances are shown in Table 1.

\section{Determination of total polyphenols concentration}

The Folin-Ciocalteu method with slight modifications was used to determine the total polyphenol concentration (Ainsworth and Gillespie, 2007). Gallic acid was used as the standard and distilled water was used as a blank. In brief, $1.0 \mathrm{~mL}$ of each diluted extract sample, in which $0.1 \mathrm{~mL}$ of extract sample was diluted to $10 \mathrm{~mL}$ by adding distilled water, punicalagin, gallic acid, and ellagic acid, was mixed with $1.0 \mathrm{~mL}$ of distilled water and $1.0 \mathrm{~mL}$ of Folin-Ciocalteu reagent and then $2 \mathrm{~mL}$ of sodium carbonate solution $(10 \%)$ was added. Absorbance was read at $765 \mathrm{~nm}$ after $60 \mathrm{~min}$ of reaction at $30^{\circ} \mathrm{C}$. Results were expressed as milligrams gallic acid equivalent per gram of pomegranate extract. Each extract sample was tested in triplicate.

\section{Determination of DPPH radical scavenging capacity}

DPPH radical scavenging capacity was determined according to the method described by Negi and Jayaprakasha (2003) with slight modifications. In brief, $2.0 \mathrm{~mL}$ of each diluted extract sample, in which $0.1 \mathrm{~mL}$ of the extract sample was diluted in $10 \mathrm{~mL}$ of distilled water, punicalagin, gallic acid, and ellagic acid, was mixed with $2.0 \mathrm{~mL}$ of DPPH-ethanolic solution. The diluted extract sample $(2.0 \mathrm{~mL})$ was mixed with ethanolic solution $(2.0 \mathrm{~mL})$. The absorbance at $517 \mathrm{~nm}$ was recorded after $10 \mathrm{~min}$. An ethanol solution was used as

Table 1. Regression equation, $R^{2}$, linear range and limit of detection (LOD) of seven substances.

\begin{tabular}{|c|c|c|c|c|}
\hline Substance & Regression equation $^{\mathrm{z}}$ & $R^{2}$ & $\begin{array}{l}\text { Linear range } \\
\left(\mathrm{mg} \cdot \mathrm{L}^{-1}\right)\end{array}$ & $\begin{array}{c}\mathrm{LOD} \\
\left(\mathrm{mg} \cdot \mathrm{L}^{-1}\right)\end{array}$ \\
\hline$\alpha$-Punicalagin & $y=36924 x-1245$ & 0.999 & $2.0-60$ & 0.23 \\
\hline$\beta$-Punicalagin & $y=20475 x-915$ & 0.999 & $2.0-60$ & 0.21 \\
\hline Gallic acid & $y=54826 x-3519$ & 0.999 & $1.5-40$ & 0.20 \\
\hline Ellagic acid & $y=10518 x-1382$ & 0.999 & $2.0-70$ & 0.19 \\
\hline Shikimic acid & $y=58903 x-22.3$ & 0.999 & $2.0-45$ & 0.18 \\
\hline 3-Dehydroshikimic acid & $y=39512 x-50.55$ & 0.999 & $2.5-50$ & 0.25 \\
\hline Pentagalloylglucose & $y=34325 x-95.66$ & 0.999 & $3.0-80$ & 0.12 \\
\hline
\end{tabular}

${ }^{\mathrm{z}} \mathrm{y}$ is the peak area; $\mathrm{x}$ refers to the concentration of each substance $\left(\mathrm{mg} \cdot \mathrm{L}^{-1}\right)$. 
a blank.

Scavenging capacity (SC) was calculated by using the following equation: $\mathrm{SC}(\%)=\left[1-\left(A_{\mathrm{i}}-A_{\mathrm{j}}\right) / A_{0}\right] \times$ 100 , where $A_{\mathrm{i}}$ is the absorbance of the extract sample mixture and DPPH-ethanolic solution, $A_{\mathrm{j}}$ is the absorbance value of the extract sample mixture and ethanolic solution, and $A_{0}$ is the absorbance value of the DPPHethanolic solution. Each extract sample was tested in triplicate.

\section{Statistical analysis}

Off-line Agilent ChemStation was used for HPLC data processing. SPSS version Rel.19.0 for Windows was used for statistical analysis. The experimental indexes value was the average value for 2015-2017. ANOVA was performed to determine the concentration difference in punicalagin and its related synthetic substances during development phases. Differences among means were considered significant at $P<0.05$ with Tukey's honest significant difference test. Correlation coefficients were determined with Pearson's correlation matrix method.

\section{Results}

Variations in punicalagin, gallic acid, and ellagic acid

The dynamic variations in punicalagin, gallic acid, and ellagic acid in different parts of two pomegranate cultivars are shown in Figure 2. The concentrations of punicalagin in the two cultivars showed similar variation patterns in the pericarp, juice, seeds, and leaves, and decreased gradually with fruit development. The peak value of punicalagin was detected in S1. This suggested that the punicalagin level was higher in the early development period and lower in the late development period. Compared with other periods, the punicalagin concentration of TSH showed a significant difference in the peel in S3, in juice in S1 and S2, and in seeds in S1 (Fig. 2A). There was a significant difference in the punicalagin concentration of TSSBT in juice in S5 (Fig. 2B). Significant differences were also found in the highest concentrations of punicalagin, gallic acid, and ellagic acid in the pericarp, juice, seeds, and leaves in S1.

The gallic acid concentrations of the two cultivars
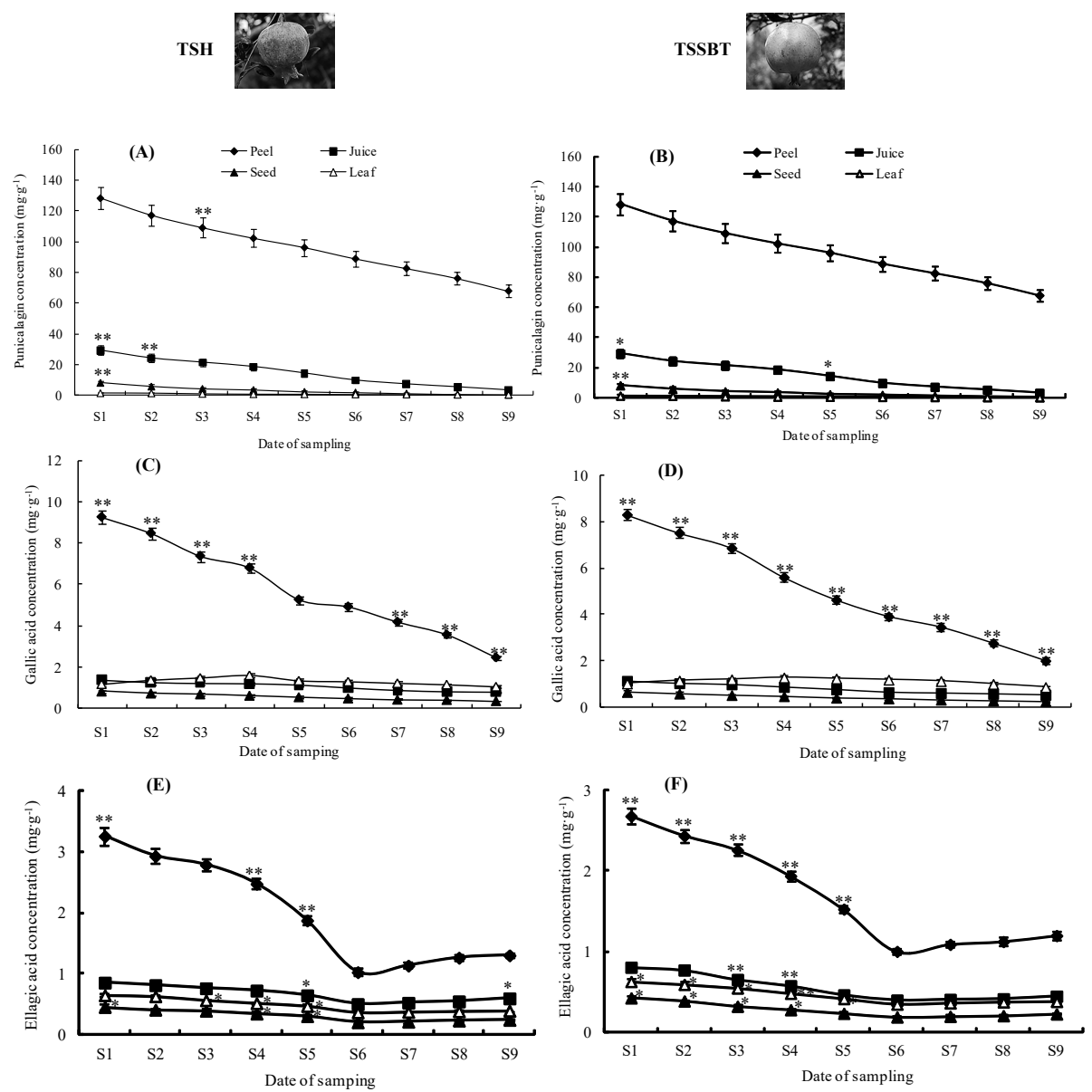

Fig. 2. Variations in punicalagin, gallic acid, and ellagic acid in different pomegranate parts during development phases. Data shown are the means of three replicates, and the vertical bar represents the standard error. * Significant difference at 0.05 levels compared to other samples in the same part in different development stages. $* *$ Significant difference at 0.01 levels compared to other samples in the same part in different development stages. 
decreased gradually in the pericarp, juice, and seeds, and the highest value was obtained in S1 (Fig. 2C, D). Gallic acid initially increased and subsequently decreased in the leaves, and its peak was observed in S4. Compared with other periods, the gallic acid concentration of TSH showed a significant difference in the peel from S1 to S4 and S7 to S9 (Fig. 2C). The difference in gallic acid in TSSBT peel was significant compared with that in other periods (Fig. 2D).

Conversely, the ellagic acid concentrations of the two cultivars initially decreased and then increased in the pericarp, juice, seeds, and leaves, and their peak values were observed in S1 and S9 (Fig. 2E, F). There was a significant difference in the ellagic acid concentrations in different parts of the two cultivars during development periods.

The concentration of punicalagin decreased in different parts in the following order: pericarp $>$ juice $>$ seeds $>$ leaves during maturation. The punicalagin concentration of TSH ranged from $79.11 \mathrm{mg} \cdot \mathrm{g}^{-1}$ to $156.74 \mathrm{mg} \cdot \mathrm{g}^{-1}$ on October 2 in the pericarp, and there was $68.12-128.46 \mathrm{mg} \cdot \mathrm{g}^{-1}$ in the TSSBT pericarp. This indicated that the punicalagin concentration in the TSH pericarp was higher than that of TSSBT. The concentration of gallic acid likewise decreased in the following order: pericarp $>$ leaves $>$ juice $>$ seeds during maturation. The concentration of ellagic acid also decreased in the following order: pericarp $>$ juice $>$ leaves $>$ seeds during maturation.

Variations in shikimic acid, 3-dehydroshikimic acid, and pentagalloylglucose

In our study, the shikimic acid concentration of the two cultivars decreased gradually in the pericarp, juice, and seeds, and the peak value appeared in S1. The concentration of shikimic acid first increased and then decreased in the leaves, and the peak was observed in S3 (Fig. 3A, B). The 3-dehydroshikimic acid concentration of the two cultivars increased gradually during development (Fig. 3C, D). The concentration of 3dehydroshikimic acid initially decreased and then increased in leaves, and the peaks were observed in S1 and S9.

Pentagalloylglucose showed a variation pattern simi-
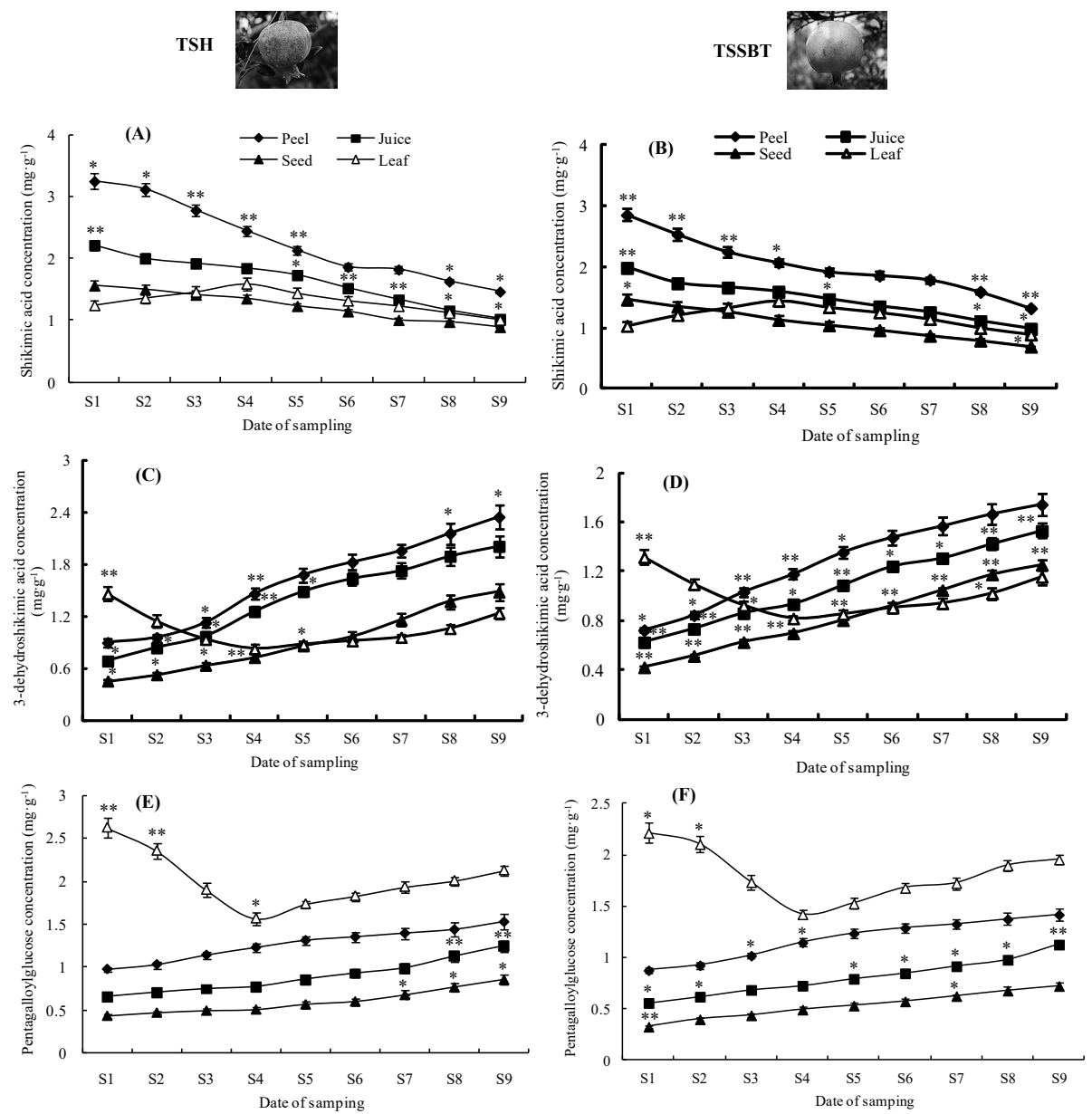

Fig. 3. Variations in shikimic acid, 3-dehydroshikimic acid, and pentagalloylglucose in different pomegranate parts during development phases. Data shown are the means of three replicates, and the vertical bar represents the standard error. * Significant difference at 0.05 levels compared to other samples in the same part in different development stages. ** Significant difference at 0.01 levels compared to other samples in the same part under different development stages. 
lar to 3-dehydroshikimic acid, and the content of the former increased gradually in the pericarp, juice, and seeds during development (Fig. 3E, F). Pentagalloylglucose initially decreased and then increased in leaves, and the peaks were detected in S1 and S9.

Significant differences were found in the concentrations of shikimic acid, 3-dehydroshikimic acid, and pentagalloylglucose in different parts in the two cultivars. The concentration of shikimic acid decreased in the following order: pericarp $>$ juice $>$ leaves $>$ seeds, except in S1 and S2. This result suggested that shikimic acid was mainly found in the pericarp. The concentration of 3-dehydroshikimic acid also decreased in the early development period (from S1 to S2): leaves $>$ pericarp $>$ juice $>$ seeds. In the late development period (from S6 to S9), the concentration of 3dehydroshikimic acid was reduced in the following order: pericarp $>$ juice $>$ seeds $>$ leaves. This finding showed that 3-dehydroshikimic acid was mainly found in the leaves and pericarp. The concentration of pentagalloylglucose likewise decreased in the following order: leaves $>$ pericarp $>$ juice $>$ seeds in different development stages.

Variations of total phenol concentration and DPPH radical scavenging activity

The total phenol concentration (Fig. 4A, B) and DPPH radical scavenging activity (Fig. 4C, D) of the two cultivars decreased gradually in the pericarp, juice, and seeds, and the peak was detected in S1. The variation pattern of total phenol content and DPPH radical scavenging activity in the leaves were different from those in the fruit. The total phenol content and DPPH radical scavenging activity in the leaves initially increased and then decreased during development. The peaks of the total phenol content and DPPH radical scavenging activity in the leaves were observed in S4.

Significant differences were found in the total phenol concentration and DPPH radical scavenging activity. The total phenol concentration and DPPH radical scavenging activity decreased in the following order: pericarp $>$ juice $>$ leaves $>$ seeds. The highest concentrations of total phenols in the TSH and TSSBT pericarps were 15.65 times and 19.88 times that in the seeds, respectively. This finding suggests that pomegranate pericarp contains high amounts of phenolic compounds and elicits strong antioxidant activities (Tzulker et al., 2007; Aviram et al., 2008).

\section{Correlation analysis}

The relationship between punicalagin and related substances was investigated through correlation analysis in the two cultivars (Table 2-5). These substances were similarly correlated in the pericarp, juice, seeds, and leaves. Punicalagin was significantly and positively correlated with gallic acid in fruit, but was not correlated with gallic acid in leaves. Gallic acid was significantly and positively correlated with ellagic acid in the fruit. Punicalagin and ellagic acid were significantly and positively correlated with shikimic acid, but significantly and negatively correlated with 3dehydroshikimic acid, in the fruit. Pentagalloylglucose
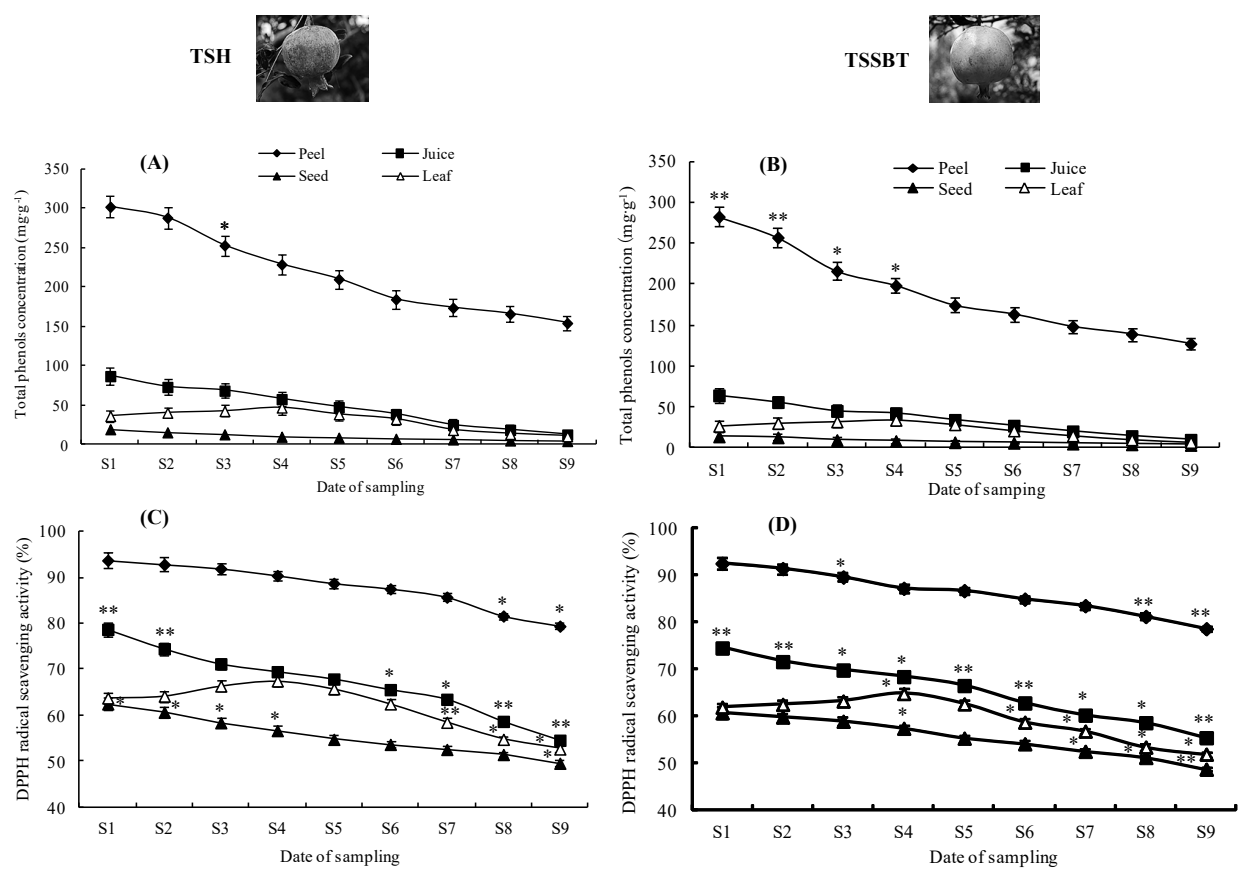

Fig. 4. Variations in total phenols concentration and DPPH radical scavenging activity in different pomegranate parts during development phases. Data shown are the means of three replicates, and the vertical bar represents the standard error. * Significant difference at 0.05 levels compared to other samples in the same part in different development stages. ** Significant difference at 0.01 levels compared to other samples in the same part in different development stages. 


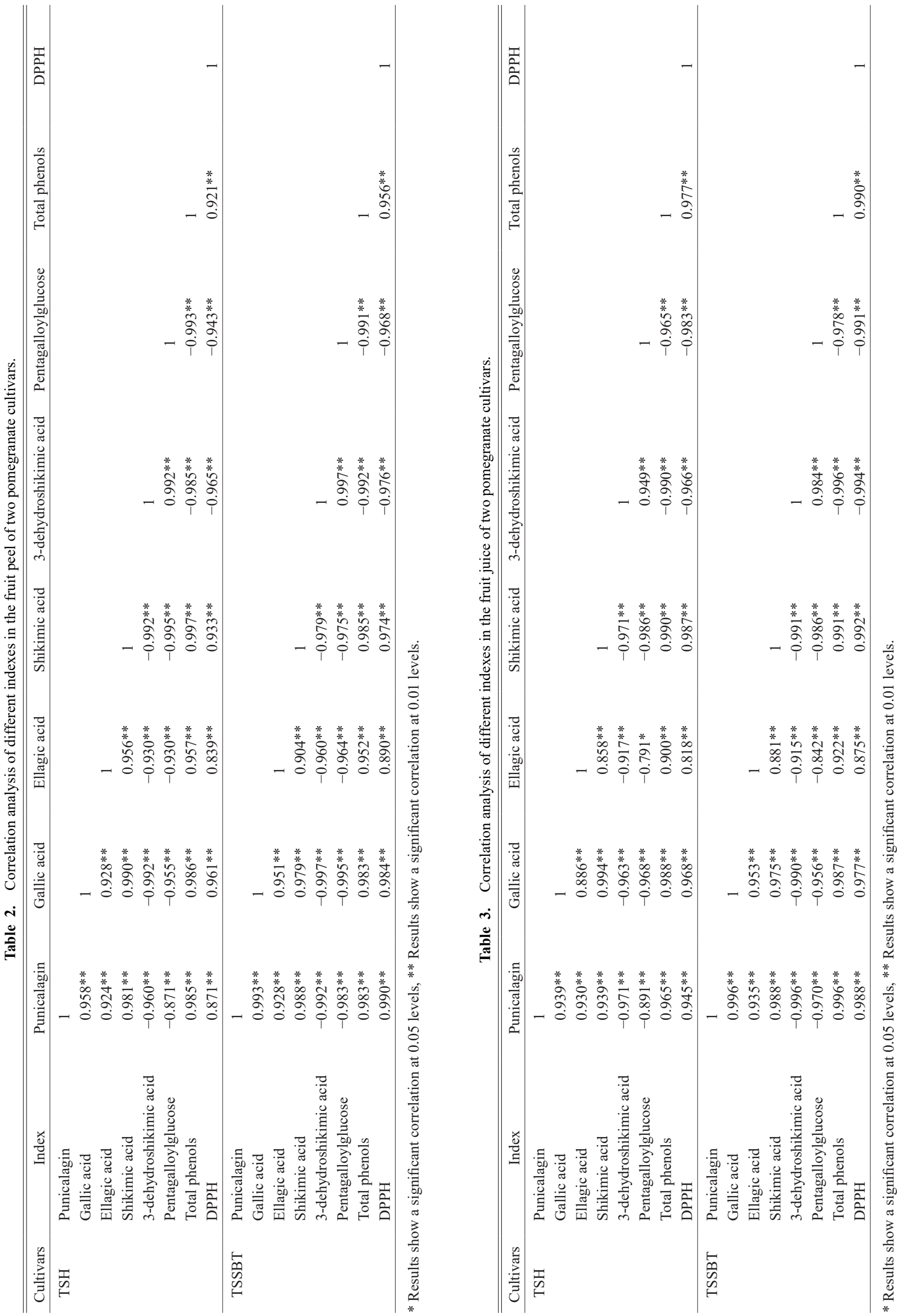




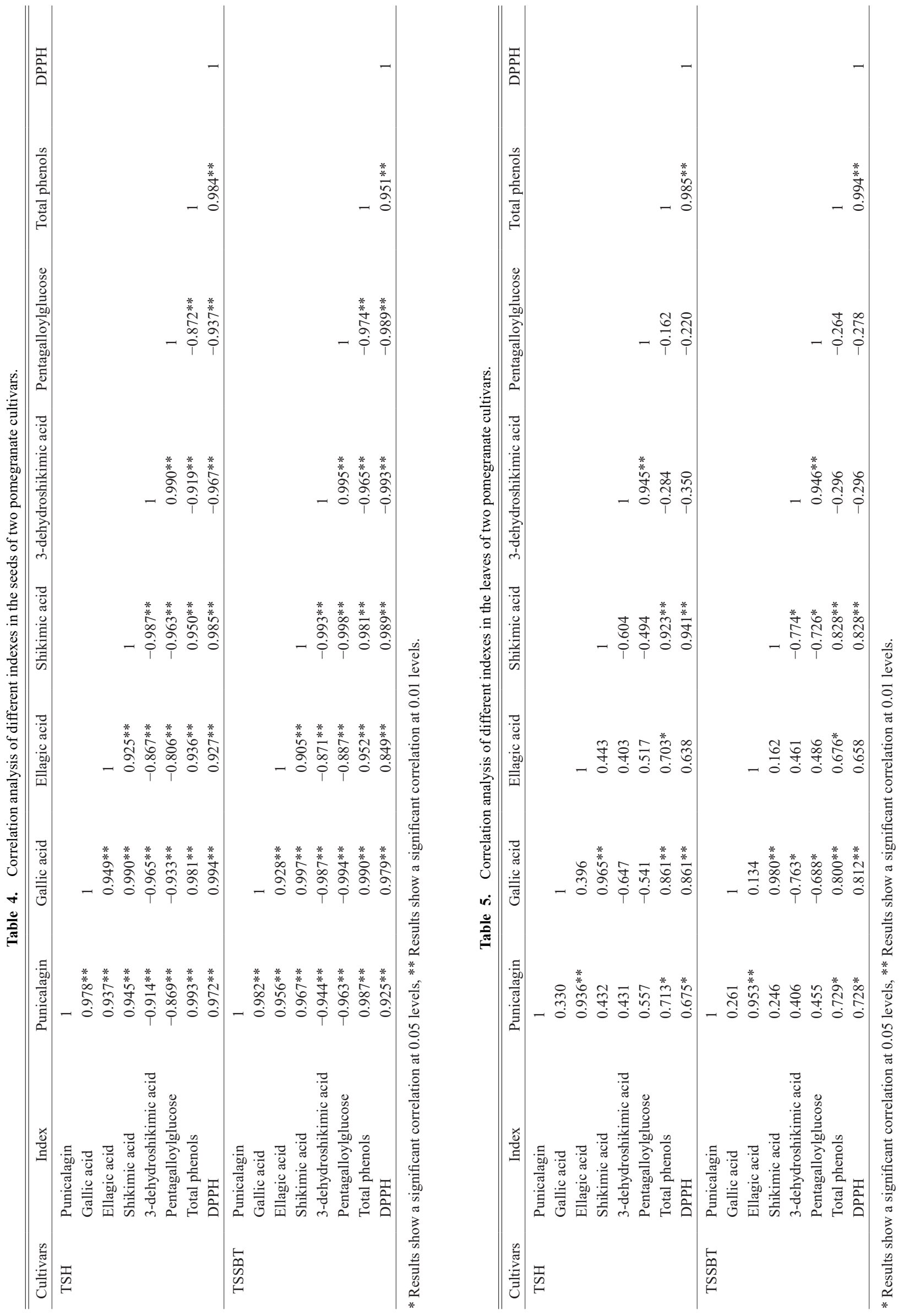


was also significantly negatively correlated with ellagic acid in the fruit.

Gallic acid was significantly and positively correlated with shikimic acid in the fruit and leaves, but was negatively correlated with 3 -dehydroshikimic acid. Shikimic acid was significantly and negatively correlated with 3-dehydroshikimic acid, and was significantly and positively correlated with total phenol content and DPPH radical scavenging activity. 3-Dehydroshikimic acid was also significantly and positively correlated with pentagalloylglucose. Pentagalloylglucose was significantly and negatively correlated with punicalagin and gallic acid in the fruit. Total phenol content was significantly and positively correlated with DPPH radical scavenging activity. Previous studies also revealed high correlations between antioxidant activity and total phenol content (Tzulker et al., 2007; Zhao et al., 2008).

\section{Discussion}

Variations in punicalagin, gallic acid, and ellagic acid

Punicalagin, gallic acid, and ellagic acid are major phenolic compounds in pomegranate. The punicalagin content decreased in different parts during maturation and this observation is consistent with that reported in Israeli pomegranate pericarp (Shwartz et al., 2009). Ellagic acid content decreased in the early development period, and this finding was in accordance with that in strawberry (Williner et al., 2003). Ellagic acid content increased in the late development period, and this was consistent with the finding in muscadine grapes (Lee and Talcott, 2004).

In our study, the punicalagin concentration of the two cultivars was highest in the pericarp. The punicalagin concentration in the pericarp in the mature stage was $40-122 \mathrm{mg} \cdot \mathrm{g}^{-1}$ (Lu et al., 2008). High punicalagin contents $\left(5.48-36.68 \mathrm{mg} \cdot \mathrm{g}^{-1}\right.$ of TSH, $3.26-29.35 \mathrm{mg} \cdot \mathrm{g}^{-1}$ of TSSBT) were also found in pomegranate juice. In previous studies, punicalagin contents were $4-565 \mathrm{mg} \cdot \mathrm{L}^{-1}$ (Fischer et al., 2011) and $20-1350 \mathrm{mg} \cdot \mathrm{L}^{-1}$ (Gil et al., 2000) in pomegranate juice. The lowest punicalagin concentration was detected in the leaves, and this finding indicated that punicalagin was not the most important phenolic compound in leaves. High punicalagin contents in the pericarp and juice have been extensively investigated. Punicalagin is also considered to be a promising multifunctional compound because of its remarkable pharmacological attributes, health-beneficial properties, bioavailability, and nontoxic nature (Kulkarni et al., 2007).

In four pomegranate parts, the concentrations of punicalagin, gallic acid, and ellagic acid significantly differed. The highest concentrations of punicalagin, gallic acid, and ellagic acid were mainly found in the pericarp. This finding suggests that phenolic compound concentrations are higher in pomegranate pericarp than in other parts (Fischer et al., 2011). The highest punicalagin concentration in the two cultivars was higher than those of gallic acid and ellagic acid in the peel. This result is in agreement with previous studies (Tzulker et al., 2007), which revealed that punicalagin was the most abundant phenolic compound in pomegranate pericarp.

Variations in shikimic acid, 3-Dehydroshikimic acid, and pentagalloylglucose

Shikimic acid is an essential compound in synthesis pathways for aromatic amino acids, alkaloids, and phenolic compounds (Gurib-Fakim, 2006). Shikimic acid is also a key intermediate for the synthesis of the neuraminidase inhibitor oseltamivir (Tamiflu), an antiinfluenza drug (Cui et al., 2014). 3-Dehydroshikimic acid is an intermediate in the catabolism of shikimic acid via the $\beta$-ketoadipate pathway (Chang et al., 2003). 3 -Dehydroshikimic acid is produced from shikimic acid via the shikimic acid pathway (Ghosh et al., 2012). Gallic acid synthesized from 3-dehydroshikimic acid has been detected in birch leaves (Ossipov et al., 2003). Our results showed that the variation pattern in shikimic acid was similar to that of gallic acid, and unlike that of 3-dehydroshikimic acid. Correlation analysis showed that gallic acid was significantly and positively correlated with shikimic acid in the fruit and leaves, but was negatively correlated with 3-dehydroshikimic acid. Shikimic acid was significantly and negatively correlated with 3-dehydroshikimic acid. These findings suggest that shikimic acid and 3-dehydroshikimic acid are closely related to gallic acid metabolism in pomegranate (Muir et al., 2011).

Pentagalloylglucose, a naturally occurring hydrolysable tannin and an active compound present in many traditional medicinal herbs, exhibits multiple biological activities (Pei et al., 2011; Kim et al., 2014). Pentagalloylglucose in the two cultivars initially decreased and then increased in the leaves. The large amount of pentagalloylglucose in pomegranate leaves was consistent with the experimental result in Tellima grandiflora (Niemetz and Gross, 2003) and Rhus typhina (Niemetz and Gross, 2001).

Gallic acid is the starting material for hydrolysable tannin synthesis (Ossipov et al., 2003), and can generate pentagalloylglucose (Grundhöfer et al., 2001). Punicalagin was significantly and positively correlated with gallic acid in the fruit. Pentagalloylglucose was significantly and negatively correlated with punicalagin and gallic acid in the fruit. Therefore, gallic acid and pentagalloylglucose are metabolized into punicalagin mainly in pomegranate fruit (Tzulker et al., 2007).

\section{Variations in total phenols and DPPH radical scaveng-} ing activity

Total phenols protect fruits from plant diseases and pests during early development, although only low amounts of phenols are required during late development (Shwartz et al., 2009). The total phenol concentra- 
tion of the two cultivars decreased gradually during development. This result is consistent with that observed in guava fruit (Bashir and Abu-Goukh, 2003). The decrease in total phenols causes a reduction in fruit astringency, which is a desirable sensory attribute of pomegranate (Kulkarni and Aradhya, 2005). The decrease in total phenols is attributed to polyphenol oxidation by polyphenol oxidase during fruit ripening (Fawole and Opara, 2013).

The antioxidant activity in different pomegranate parts has been quantified in terms of its radical scavenging potential (Kulkarni and Aradhya, 2005). DPPH radical scavenging activity was significantly and positively correlated with total phenol. Previous studies also revealed high correlations between antioxidant activity and total phenol content (Tzulker et al., 2007; Zhao et al., 2008). The antioxidant activity of pomegranate peel was the highest, followed by the juice, and was the lowest in seeds. This result is supported by a study in Turkey pomegranate (Orak et al., 2012) that revealed the levels of antioxidants in different pomegranate parts. The difference in the antioxidant activity of the peel, juice, leaves and seeds may be ascribed to their different phenolic compositions (Singh et al., 2002). As a result, pomegranate peel could be further exploited and used in health supplements.

Punicalagin, ellagic acid, gallic acid, shikimic acid, 3-dehydroshikimic acid, ellagic acid, and pentagalloylglucose are bioactive compounds in pomegranate. Total phenol content and DPPH radical scavenging activity were significantly and positively correlated with punicalagin, gallic acid, shikimic acid, ellagic acid in the fruit and leaves. These results implied that the concentrations of punicalagin, gallic acid, shikimic acid, and ellagic acid were highly correlated with total phenol content and antioxidant activity. 3-Dehydroshikimic acid and pentagalloylglucose were significantly and negatively correlated with total phenol content and DPPH radical scavenging activity in the fruit. This phenomenon may be influenced by development stage and different pomegranate parts.

The seasonal variations in punicalagin and related synthetic compounds during development phases in different pomegranate parts were investigated. Further studies should be performed to understand the metabolism of punicalagin in pomegranate. The relationship of punicalagin and related regulatory enzymes should also be further explored. Punicalagin regulatory enzyme genes should be cloned, and their expression levels during development should be analyzed at the molecular level.

\section{Conclusions}

Punicalagin and related substances showed different variation patterns in various pomegranate parts during development. Punicalagin was the most abundant phenolic compound in the pericarp; it was significantly positively correlated with gallic acid and shikimic acid in the fruit, and significantly negatively correlated with pentagalloylglucose and 3-dehydroshikimic acid. Pomegranate pericarp contained abundant phenolic compounds and exhibited strong antioxidant activity. Our study revealed the variation patterns and tissue differences of punicalagin content and related substances in pomegranate. This could provide a theoretical basis for the development and utilization of different pomegranate parts.

\section{Acknowledgements}

The authors thank the group at the Shandong Institute of Pomology for their contribution to some field trials.

\section{Literature Cited}

Ainsworth, E. A. and K. M. Gillespie. 2007. Estimation of total phenolic content and other oxidation substrates in plant tissues using Folin-Ciocalteu reagent. Nat. Protoc. 2: 875-877.

Aviram, M., N. Volkova, R. Coleman, M. Dreher, M. K. Reddy, D. Ferreira and M. Rosenblat. 2008. Pomegranate phenolics from the peels, arils, and flowers are antiatherogenic: studies in vivo in atherosclerotic apolipoprotein E-deficient (E0) mice and in vitro in cultured macrophages and lipoproteins. J. Agr. Food Chem. 56: 1148-1157.

Bashir, H. A. and A. B. A. Abu-Goukh. 2003. Compositional changes during guava fruit ripening. Food Chem. 80: 557563.

BenSaad, L. A., K. H. Kim, C. C. Quah, W. R. Kim and M. Shahimi. 2017. Anti-inflammatory potential of ellagic acid, gallic acid and punicalagin A\&B isolated from Punica granatum. BMC. Complem. Altern. M. 17: 47-57.

Chang, Y. C., E. A. Almy, G. A. Blamer, J. I. Gray, J. W. Frost and G. M. Strasburg. 2003. Antioxidant activity of 3dehydroshikimic acid in liposomes, emulsions, and bulk oil. J. Agr. Food Chem. 51: 2753-2757.

Chater, J. M., D. J. Merhaut, Z. Jia, P. A. Mauk and J. E. Preece. 2018. Fruit quality traits of ten California-grown pomegranate cultivars harvested over three months. Sci. Hortic. 237: $11-19$.

Clementi, M. E., G. Pani, B. Sampaolese and G. Tringali. 2017. Punicalagin reduces $\mathrm{H}_{2} \mathrm{O}_{2}$-induced cytotoxicity and apoptosis in PC12 cells by modulating the levels of reactive oxygen species. Nutr. Neurosci. 21: 1-8.

Cui, Y. Y., C. Ling, Y. Y. Zhang, J. Huang and J. Z. Liu. 2014. Production of shikimic acid from Escherichia coli through chemically inducible chromosomal evolution and cofactor metabolic engineering. Microb. Cell Fact. 13: 21. DOI: 10.1186/1475-2859-13-21.

Dewick, P. M. 2009. Medicinal Natural Products: A Biosynthetic Approach, 3rd Edition. p. 138-150. Jone Wiley \& Sons, Ltd.

Fawole, O. A. and U. L. Opara. 2013. Changes in physical properties, chemical and elemental composition and antioxidant capacity of pomegranate (cv. Ruby) fruit at five maturity stages. Sci. Hortic. 150: 37-46.

Feng, L. J., Y. L. Yin, Y. M. Fang and X. M. Yang. 2017. Quantitative determination of punicalagin and related substances in different parts of pomegranate. Food Anal. Methods 10: 3600-3606.

Fischer, U. A., R. Carle and D. R. Kammerer. 2011. Identification and quantification of phenolic compounds from pomegranate (Punica granatum L.) peel, mesocarp, aril and differently 
produced juices by HPLC-DAD-ESI/MSn. Food Chem. 127: 807-821.

Ghosh, S., Y. Chisti and U. C. Banerjee. 2012. Production of shikimic acid. Biotechnol. Adv. 30: 1425-1431.

Gil, M. I., F. A. Tomás-Barberán, B. Hesspierce, D. M. Holcroft and A. A. Kader. 2000. Antioxidant activity of pomegranate juice and its relationship with phenolic composition and processing. J. Agr. Food Chem. 48: 4581-4589.

Grundhöfer, P., R. Niemetz, G. Schilling and G. G. Gross. 2001. Biosynthesis and subcellular distribution of hydrolyzable tannins. Phytochemistry 57: 915-927.

Gurib-Fakim, A. 2006. Medicinal plants: traditions of yesterday and drugs of tomorrow. Mol. Aspects Med. 27: 1-93.

Kim, T. W., S. Paveen, Y. H. Lee and S. L. Yong. 2014. Comparison of cytotoxic effects of pentagalloylglucose, gallic acid, and its derivatives against human cancer MCF-7 and MDA MB-231 cells. B. Korean Chem. Soc. 35: 987-988.

Kim, Y. E., C. J. Hwang, H. P. Lee, C. S. Kim, D. J. Son, Y. W. Ham, M. Hellström, S. B. Han, H. S. Kim, E. K. Park and J. T. Hong. 2017. Inhibitory effect of punicalagin on lipopolysaccharide-induced neuroinflammation, oxidative stress and memory impairment via inhibition of nuclear factor-kappab. Neuropharmacology 117: 21-32.

Kulkarni, A. P. and S. M. Aradhya. 2005. Chemical changes and antioxidant activity in pomegranate arils during fruit development. Food Chem. 93: 319-324.

Kulkarni, A. P., H. S. Mahal, S. Kapoor and S. M. Aradhya. 2007. In vitro studies on the binding, antioxidant, and cytotoxic actions of punicalagin. J. Agr. Food Chem. 55: 1491-1500.

Landete, J. M. 2011. Ellagitannins, ellagic acid and their derived metabolites: a review about source, metabolism, functions and health. Food Res. Int. 44: 1150-1160.

Lee, J. H. and S. T. Talcott. 2004. Fruit maturity and juice extraction influences ellagic acid derivatives and other antioxidant polyphenolics in muscadine grapes. J. Agr. Food Chem. 52: 361-366.

Lu, J., K. Ding and Q. Yuan. 2008. Determination of punicalagin isomers in pomegranate husk. Chromatographia 68: 303-306.

Modaeinama, S., M. Abasi, M. M. Abbasi and R. Jahanbanesfahlan. 2015. Antitumoral properties of punica granatum (pomegranate) peel extract on different human cancer cells. Asian Pac. J. Cancer. Pre. 16: 5697-5701.

Muir, R. M., A. M. Ibáñez, S. L. Uratsu, E. S. Ingham, C. A. Leslie, G. H. Mcgranahan, N. Batra, S. Goyal, J. Joseph, E. D. Jemmis and A. M. Dandekar. 2011. Mechanism of gallic acid biosynthesis in bacteria (Escherichia coli) and walnut (Juglans regia). Plant Mol. Biol. 75: 555-565.

Negi, P. S. and G. K. Jayaprakasha. 2003. Antioxidant and antibacterial activities of Punica granatum peel extracts. J. Food Sci. 68: 1473-1477.

Niemetz, R. and G. G. Gross. 2001. Gallotannin biosynthesis: $\beta$ glucogallin: hexagalloyl 3-O-galloyltransferase from Rhus typhina leaves. Phytochemistry 58: 657-661.

Niemetz, R. and G. G. Gross. 2003. Oxidation of pentagalloylglucose to the ellagitannin, tellimagrandin II, by a phenol oxidase from Tellima grandiflora leaves. Phytochemistry 62: 301-306.

Orak, H. H., H. Yagar and S. S. Isbilir. 2012. Comparison of antioxidant activities of juice, peel, and seed of pomegranate (Punica granatum L.) and inter-relationships with total phenolic, tannin, anthocyanin, and flavonoid contents. Food Sci. Biotechnol. 21: 373-387.

Orgil, O., E. Schwartz, L. Baruch, I. Matityahu, J. Mahajna and R. Amir. 2014. The antioxidative and anti-proliferative potential of non-edible organs of the pomegranate fruit and tree. LWT-Food. Sci. Technol. 58: 571-577.

Ossipov, V., J. P. Salminen, S. Ossipova, E. Haukioja and K. Pihlaja. 2003. Gallic acid and hydrolysable tannins are formed in birch leaves from an intermediate compound of the shikimate pathway. Biochem. Syst. Ecol. 31: 3-16.

Pei, Y., Z. P. Chen, H. Q. Ju, M. Komatsu, Y. H. Ji, G. Liu, C. Guo, Y. Zhang, C. Yang, Y. Wang and K. Kitazato. 2011. Autophagy is involved in anti-viral activity of pentagalloylglucose (PGG) against Herpes simplex virus type 1 infection in vitro. Biochem. Bioph. Res. Co. 405: 186-191.

Qin, G., C. Xu, R. Ming, H. Tang, R. Guyot, E. M. Kramer, Y. Hu, X. Yi, Y. Qi, X. Xu, Z. Gao, H. Pan, J. Jian, Y. Tian, Z. Yue and Y. Xu. 2017. The pomegranate (Punica granatum L.) genome and the genomics of punicalagin biosynthesis. Plant J. 91: 1108-1128.

Schieber, A., P. Keller and R. Carle. 2001. Determination of phenolic acids and flavonoids of apple and pear by highperformance liquid chromatography. J. Chromatography A. 910: 265-273.

Seeram, N. P., L. S. Adams, S. M. Henning, Y. T. Niu, Y. J. Zhang, M. G. Nair and D. Heber. 2005. In vitro antiproliferative, apoptotic and antioxidant activities of punicalagin, ellagic acid and a total pomegranate tannin extract are enhanced in combination with other polyphenols as found in pomegranate juice. J. Nutr. Biochem. 16: 360-367.

Shwartz, E., I. Glazer, I. Bar-Ya'akov, I. Matityahu, I. Bar-Ilan, D. Holland and R. Amir. 2009. Changes in chemical constituents during the maturation and ripening of two commercially important pomegranate accessions. Food Chem. 115: 965-973.

Singh, M., A. Jha, A. Kumar, N. Hettiarachchy, A. K. Rai and D. Sharma. 2014. Influence of the solvents on the extraction of major phenolic compounds (punicalagin, ellagic acid and gallic acid) and their antioxidant activities in pomegranate aril. J. Food Sci. Technol. 51: 2070-2077.

Singh, R. P., K. N. Chidambara Murthy and G. K. Jayaprakasha. 2002. Studies on the antioxidant activity of pomegranate (Punica granatum) peel and seed extracts using in vitro models. J. Agric. Food Chem. 50: 81-86.

Tang, J., B. Li, S. Hong, C. Liu, J. Min, M. Hu, Y. Li and L. Hong. 2017. Punicalagin suppresses the proliferation and invasion of cervical cancer cells through inhibition of the $\beta$ catenin pathway. Mol. Med. Rep. 16: 1439-1444.

Tzulker, R., I. Glazer, I. Bar-Ilan, D. Holland, M. Aviram and R. Amir. 2007. Antioxidant activity, polyphenol content and related compounds in different fruit juices and homogenates prepared from 29 different pomegranate accessions. J. Agr. Food Chem. 55: 9559-9570.

Vermerris, W. and R. Nicholson. 2006. Phenolic compound biochemistry. P. 130-134. Netherlands: Verlag New York Inc.

Williner, M. R., M. E. Pirovani and D. R. Güemes. 2003. Ellagic acid content in strawberries of different cultivars and ripening stages. J. Sci. Food Agr. 83: 842-845.

Xie, J., Z. Zhang, S. Liang, C. Yang, C. You and Y. Li. 2013. Simultaneous determination of the 11 contents in the combination extracts of Rhei Radix et Rhizoma and Moutan Cortex by HPLC wavelength switching method. Chinese J. Pharma. Anal. 33: 103-107.

Zhao, H., W. Fan, J. Dong, J. Lu, J. Chen, L. Shan, Y. Lin and W. Kong. 2008. Evaluation of antioxidant activities and total phenolic contents of typical malting barley varieties. Food Chem. 107: 296-304.

Zhou, N., C. Xia, H. Zhang, Y. Zhang and G. Liu. 2010. Determination of shikimic acid in Pinus armandi by HPLC. J. Dali Univ. 9: 4-15, 18. 